\section{IUPAC Periodic Table of the Elements and Isotopes (IPTEI) for the Education Community (IUPAC Technical Report)}

Norman E. Holden, Tyler B. Coplen, John K. Böhlke, Lauren V. Tarbox, Jacqueline Benefield, John R. de Laetera, Peter G. Mahaffy, Glenda O'Connorb, Etienne Rotha, Dorothy H. Tepper, Thomas Walczyk, Michael E. Wieser and Shigekazu Yoneda

Pure and Applied Chemistry 2018

Volume 90, Issue 12, pp. 1833-2092

The IUPAC Periodic Table of the Elements and Isotopes (IPTEI) was created to familiarize students, teachers, and non-professionals with the existence and importance of isotopes of the chemical elements. The IPTEI is modeled on the familiar Periodic Table of the Chemical Elements. The IPTEI is intended to hang on the walls of chemistry laboratories and classrooms. Each cell of the IPTEI provides the chemical name, symbol, atomic number, and standard atomic weight of an element. Color-coded pie charts in each element cell display the stable isotopes and the relatively long-lived radioactive isotopes having characteristic terrestrial isotopic compositions that determine the standard atomic weight of each element. The background color scheme of cells categorizes the 118 elements into four groups: (1) white indicates the element has no standard atomic weight, (2) blue indicates the element has only one isotope that is used to determine its standard atomic weight, which is given as a single value with an uncertainty, (3) yellow indicates the element has two or more isotopes that are used to determine its standard atomic weight, which is given as a single value with an uncertainty, and (4) pink indicates the element has a well-documented variation in its atomic weight, and the standard atomic weight is expressed as an interval. An element-by-element review accompanies the IPTEI and includes a chart of all known stable and radioactive isotopes for each element. Practical applications of isotopic measurements and technologies are included for the following fields: forensic science, geochronology, Earth-system sciences, environmental science, and human health sciences, including medical diagnosis and treatment.

\section{https://doi.org/10.1515/pac-2015-0703}

\section{On the Discovery of New Elements (IUPAC/IUPAP Provisional Report)}

Sigurd Hofmann, Sergey N. Dmitriev, Claes

Fahlander, Jacklyn M. Gates, James B. Roberto and Hideyuki Sakai

Pure and Applied Chemistry 2018

Volume 90, Issue 11, pp. 1773-1832

Almost thirty years ago the criteria that are currently used to verify claims for the discovery of a new element were set down by the comprehensive work of a Transfermium Working Group, TWG, jointly established by IUPAC and the International Union of Pure and Applied Physics (IUPAP). The recent completion of the naming of the 118 elements in the first seven periods of the Periodic Table of the Elements was considered as an opportunity for a review of these criteria in the light of the experimental and theoretical advances in the field. In late 2016 the Unions decided to establish a new Joint Working Group, JWG, consisting of six members determined by the Unions. A first meeting of the JWG was in May 2017. One year later this report was finished. In a first part the works and conclusions of the TWG and the Joint Working Parties, JWP, decisions on the discovery of the now named elements are summarized. Possible experimental developments for production and identification of new elements beyond the presently known ones are estimated. Criteria and guidelines for establishing priority of discovery of these potential new elements are presented. Special emphasis is given to a description for the application of the criteria and the limits for their applicability.

In accordance with the Terms of Reference under which the JWG was established, this report has been provisionally accepted by IUPAC and IUPAP, and is open for comment for 5 months. Comments should be directed to Zhou Qifeng <qfzhou@iupac.org>, President of IUPAC and to Kennedy Reed <reed5@llnl. gov>, President of IUPAP, and will be accepted up to 31 March 2019.

https://doi.org/10.1515/pac-2018-0918 